\title{
Fault Diagnosis of the Bearing with Low Speed and High Load
}

\author{
Jiahua Han a, Wen Yan, Jinhua Cao \\ Mechanical Engineering College, Shijiazhuang 050003, China \\ athjh1025@163.com
}

Keywords: Low Speed, High-Loaded, Fault Problems, Diagnosis Methods.

\begin{abstract}
As the core component of large-scale machine, the performance of low speed high load bearings directly affect the operation of the machine, the fault diagnosis methods of the bearings has been a hot issue. By list different types of fault problems, at the same time, there are many methods of such bearings in recent years, the classification and comparative analysis of fault diagnosis method in the paper, and finally make a summary and outlook.
\end{abstract}

\section{Introduction}

As large engineering components, low speed high load bearings often used in such as cranes, converter and other large mechanical devices. From the structural characteristics and installation layout of these bearings, it is difficult to carry out disintegration detection, and according to the survey, low speed high load bearings generally have a long service life and bear a large load, so they are one of the high-failure parts of large machine. Bearings have a rated life according to their characteristics, but in most cases the bearings are not rated for the different life of the corresponding failure, low speed high load bearings as the core components of large parts, because the failure will cause greater Economic losses, so there are many people pay attention to this type of bearing fault diagnosis .Aiming at the characteristics of the fault frequency in the low frequency region, complicated speed variation and low speed and heavy load, many corresponding fault diagnosis methods have emerged at home and abroad. This paper gives a brief overview.

\section{Fault types of low speed high load bearings}

The basic structure of low speed high load bearings is similar to ordinary bearings, consisting of inner ring, outer ring, rolling element and cage. Low speed high load bearings are characterized by low running speed, high load, long service life, dismantling repair complex, as well as lubrication and other cau ses more prone to failure of bearings, shorten the service life, affecting the normal operation of large machinery. According to statistics, 30\% of large-scale rotating machinery failure due to damage caused by low speed high load bearings.

According to the structure, there are four types of faults: inner ring failure, outer ring failure, rolling element failure and cage failure. According to the number of damage, it is divided into many faults caused by single bearing failure and multiple bearing damage, According to the failure mode is divided into: bearing components due to poor working environment due to wear and tear caused by failure, long-term high-load operation fatigue failure, due to air, rain corrosion failure, due to excessive load or improper assembly caused by the impact of fracture failure, Due to improper lubrication caused by abrasion and other failure modes is the most common type of bearings.

\section{Low speed ,high-loaded bearings fault detection technology development}

\subsection{Wavelet transform}

Large machine bearings often located in the internal of machine, so this is not convenient to extract fault signal feature [1], for this reason, there often using installed sensors at external of machine, using this approach collect feature signals performance confused, it difficult find fault characteristics, through wavelet transform can gain obvious feature curve [2-3].Wavelet transform is a powerful tool to processing non-stationary signals. It can enlarge the local characteristics of the signal and has the 
characteristics of filter. [4-5] wavelet transform main transform the sensors collect noise signals into time and frequency domain, bearings wear and tear and fault make noise signal increase high frequency band, then through wavelet transform extract appropriate high frequency band for envelope spectrum analysis.

\subsection{Acoustic Emission}

Low speed, heavy load bearing is the most widely used in large machine. In recent years, unexpected bearing failures have frequently appeared, variable speed caused their fast rising voltage pulses and high switching frequencies, bearings repeated discharging can gradually breakdown the bearing raceways. In order to solve the problems, acoustic emission come into being. Acoustic emission is material accept outside load or internal force [6], through elastic wave release strain phenomenon. There are two conditions when using acoustic emission: first is material bear load, second is material internal structure uneven or have faults. When acoustic emission apply to bearings diagnosis often using fig. 1 [7].

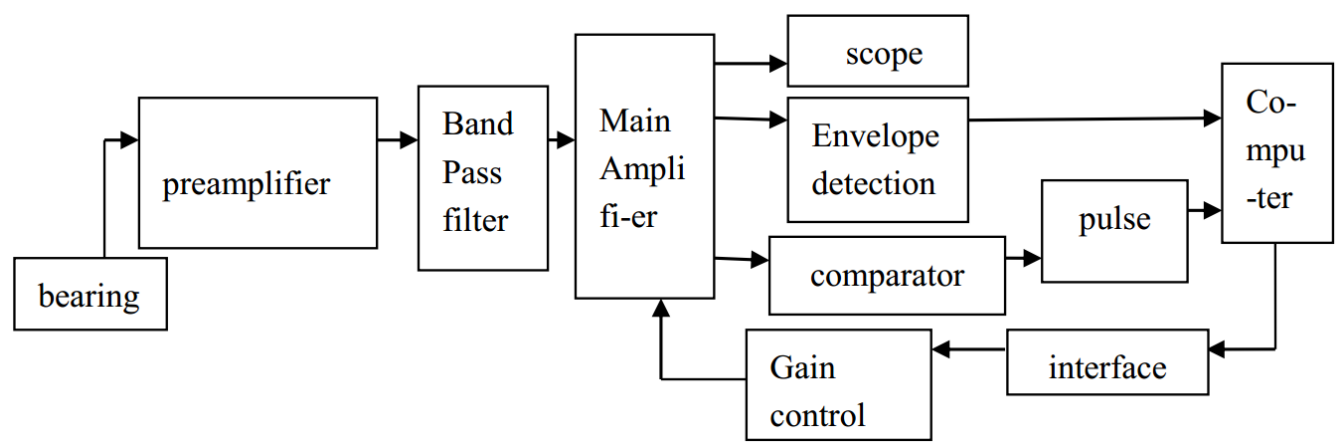

Fig. 1 Bearing acoustic emission test system

\subsubsection{Acoustic emission signal analysis}

Yoshioka and Fujiwara [8] compared the acoustic emission and vibration methods in an accelerated rolling contact fatigue experiment, and concluded that the acoustic emission could forecast the time of crack earlier. Hawman and Galinaitis [9] compared the acoustic emission with vibration signals under different crack sizes, and showed that the AE-based method can detect smaller crack size more effective. [10] Fault features are extracted by high frequency resonation method and frequency spectrum in the AE signals. Through the fault features analysis the bearings fault, the main amplifier output three types of signals, first is acoustic emission is original signal, second is envelope detection signal, third is pulse signal. There main use envelope signal, compare with original signal, it belong low frequency suitable for low speed bearings and heavy loads so it more easy to processing data and $\mathrm{A} / \mathrm{D}$ transform.

\subsection{Resonant demodulation}

Bearings produce vibration have two conditions, first is bearings rolling components surface cause random vibration, second is external forces or faults cause bearing components produce vibration on the natural frequency. When bearings normal operation there is low vibration, when there is faults, there have obvious resonance, it is related to bearing component relatively speed, then, extract high frequency band for research, through band-pass filter separate the nature frequency, filter high frequency vibration gain only have low frequency fault signals, through observe the signal frequency, judge bearings position and features.

\subsubsection{Acceleration sensor principle}

Sensor is cantilever beam structure, when it bear impact, cantilever beam produce free attenuation at internal of shell, nature frequency of sensors have difference when it assemble different position. On actual measurement, using hammer strike method record resonance frequency of sensors, for low speed, high-loaded bearings the process of resonant demodulation at fig.2 [11]. 


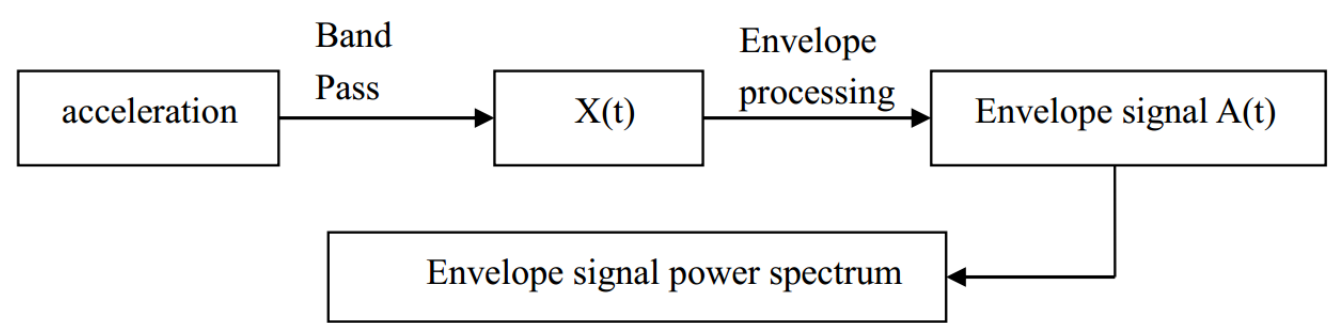

\subsection{Finite element analysis}

Fig. 2 Flow chart of analysis to resonant demodulation

Finite element analysis is mainly through the establishment of finite element model, its static contact analysis to determine the stress and deformation of the various components of the law [1213]. For the research of low speed and heavy load bearing, firstly, the contact relationship between the components of the bearing is determined, and then the corresponding algorithm is selected to analyze the finite element and analyze the statics [14] Outer ring, rolling element, the cage between the stress calculation to determine the deformation of the various parts of the law, and then analyze the main causes of deformation.

\section{Conclusion and prospect}

For the fault diagnosis of low speed heavy-load bearings, with the continuous emergence of diagnostic methods, such fault diagnosis technology has made great progress, many methods have been put into practical use, the actual effect is obvious, but some technology is still in the experimental stage, Key issues remain to be resolved. Overall, low speed high load bearing fault theory and applications also need to continue to improve. The development trend of this kind of bearing fault diagnosis technology:

1) Perfect shock pulse method, wavelet analysis, resonance demodulation method based on traditional time domain, frequency domain analysis technology and theory.

2) Researching on the operation law, bearing condition and structure characteristic of low speed and heavy load bearing, this paper studies the fault diagnosis technology of this kind of bearing.

3) To explore the new method of fault diagnosis of modern artificial intelligence, combined with low-speed heavy-load bearing fault problem, on the basis of artificial intelligence diagnosis of such bearings, the new method will be the future diagnosis of a long time development direction and research focus.

4) In view of the advantages and disadvantages of fault diagnosis methods, in order to fully and accurately reflect the characteristics of bearing failure, different fault diagnosis methods will be used in combination to make full use of their advantages will remain one of the research focus and technical path.

\section{References}

[1] Xue Y J, Cao J X, Tian R F, et al. Feature extraction of bearing vibration signals using autocorrelation denoising and improved Hilbert-Huang transformation. International Journal of Digital Content Technology and Its Application. Vol. 6 (2012) No. 4, p. 150-158.

[2] Lv Chen, Gui Zeng, Ye Hao, et al. Abrasion fault diagnosis of main bearing based on noise and wavelet envelope spectrum.CENT. SOUTH UNIV. TECHNOL. Vol. 34 (2003) No. 4, p.460462.

[3] Tse P W, Peng Y H, Yam R. Wavelet analysis and envelope detection for rolling element bearing fault diagnosis their affectivities and flexibilities. Journal of Vibration and Acoustics. Vol. 123 (2001) No. 1, p. 303-310.

[4] Wen Cheng Yu, Dong Liang, Jin Xin. Feature Extraction of Bearing Vibration Signals Using Second Generation Wavelet and Spline-Based Local Mean Decomposition. Shanghai Jiaotong University and Springer-Verlag Berlin Heidelberg. Vol. 20 (2015) No.1, p. 56-60.

[5] HOU Shu- min, LI You- rong, LIU Guang- lin, Low Speed Rolling Bearing Fault Diagnosis 
Based on General Harmonic Wavelet. Coal Mine Machinery. Vol.12 (2005) p.53-155.

[6] Pandya, D.H., Upadhyay, S.H., Harsha, S.P. Fault Diagnosis of Rolling Element Bearing with Intrinsic Mode Function of Acoustic Emission Data using AFP-KNN. Expert Syst.Appl.2013, p. 4137-4145.

[7] Gan xiao ye, Kou hui. Low speed heavy load large bearing fault characteristics and diagnosis discussion. Plant Maintenance Engineering. Vol (1998) No.2, p. 12-16.

[8] Yoshioka, T., Fujiwara, T. Measurement of Propagation Initiation and Propagation Time of Rolling Contact Fatigue Crack by Observation of Acoustic Emission and Vibration. Tribology Series Vol.12 (1987) p. 29-33.

[9] Hawman, M.W, Galinaitis, W.S.: Acoustic Emission Monitoring of Rolling Element Bearing. In: Proceedings of IEEE Ultrasonics Symposium. Chicago, 1998, p. 885-889.

[10]Phuong Nguyen, Myeongsu Kang, Jaeyoung Kim. Reliable Fault Diagnosis of Low-Speed Bearing Defects Using a Genetic Algorithm. PRICAI 2014, LNAI 8862, 2014, p. 248-255.

[11] Wang Zhi Gang, Li You rong, Zhu Rui sun, Lu Yong. A new demodulated resonance method for fault diagnosis of low speed and high load rolling bearing, Coal Mine MachineryVol. 8 (2002) p. 74-76.

[12] Wang Xingdong, Dong Yuanlong, Liu Yuanjiong. Analysis and study of low speed, heavy load rolling bearings with finite element method. J.of Wuhan Uni.of Sci. \& Tech. (Natural Science Edition). Vol. 31 (2008) No. 1, p.105-107.

[13] MENG Fanming. Three-dimensional finite element analysis for thermo-elasto-hydrodynamic performances for a water Lubricated bearing system.Journal of Chongqing University. Vol. 36 (2013) No.2, p. 121-126.

[14] WANG Shilong, WANG Xia, ZHOU Jie, et al. Compaction mechanism oriented axis structure and size design and finite element method checking. Journal of Chongqing University. Vol. 37 (2014) No. 8, p. 12-18. 